\title{
Some Uniqueness Results of the Solutions for Two Kinds of Riccati Equations with Variable Fractional Derivative
}

\author{
Shi-you Lin $(\mathbb{D}$, Li-sha Chen $(\mathbb{D}$, and Bo-yang Li $(\mathbb{D}$ \\ Key Laboratory of Data Science and Smart Education, Ministry of Education, and School of Mathematics and Statistics, \\ Hainan Normal University, Haikou, Hainan 571158, China \\ Correspondence should be addressed to Shi-you Lin; linsy1111@foxmail.com
}

Received 4 December 2021; Accepted 20 December 2021; Published 17 January 2022

Academic Editor: Jingshi Xu

Copyright (C) 2022 Shi-you Lin et al. This is an open access article distributed under the Creative Commons Attribution License, which permits unrestricted use, distribution, and reproduction in any medium, provided the original work is properly cited.

\begin{abstract}
We mainly discuss the relevant properties of the solution of the Riccati equation in this paper. By virtue of our previous derivation, we will give several uniqueness conclusions that the initial value problems of some kinds of the Riccati equation with variable fractional derivative are solved under certain conditions. Additionally, we also point out an error in the work of Kashkari and Syam and correct it.
\end{abstract}

\section{Introduction}

In recent years, properties of solutions for initial value problems of fractional differential equations have been widely studied. With the emergence of the Riccati equation in more and more scientific fields, such as atmospheric science, physics, control science, and engineering, see refs. [1-3], it is particularly urgent to study the qualitative theory of the solution of this type of equation. As everyone knows, the form of Riccati equation with constant fractional order derivative is described as follows:

$$
D^{\alpha} w(u)=l(u) w^{2}(u)+m(u) w(u)+n(u)
$$

where $\alpha$ is a constant which belongs to the open interval $(0,1)$ and the functions $l(u), m(u), n(u) \in C[0,1]$. Many achievements have been made in the research on the solution of the initial value problem of this type of equation, see refs. [4-11]. Among them, we mention that Kashkari et al. in ref. [4] gave the uniqueness and existence conclusions under the Lipschitz condition; Khader et al. in ref. [5] used the fractional Chebyshev finite difference method to solve the approximate solution; in 2019, Yan et al. in ref. [6] studied the uniqueness and existence issue by applying the spectral collocation method and Banach fixed point theorem, etc. However, so far, as we know, there are few relevant study results on the cases with variable fractional derivative, and most of them focus on the solutions of specific examples. For example, Baleanu et al. in ref. [11] used the spectral collocation method to find the approximate solution; Syam et al. in ref. [12] used the reproducing kernel Hilbert space method to solve the equation and obtained the uniform convergence and the existence of the approximate solution. The work of Tvyordyj et al. in 2021 (see ref. [13]) is mainly to calculate the numerical solution. Therefore, we adopt here a study method which differs from the above literature and derive the uniqueness of the solution of this issue under some reasonable assumptions, without seeking the exact or approximate solution. More specifically, the first kind initial value problem, we mainly study in this paper is stated as below:

$$
\left\{\begin{array}{l}
D^{\alpha(u)} w(u)=l(u) w^{2}(u)+n(u), \quad u \in(0,1) \\
w(0)=0
\end{array}\right.
$$

where $l(u)$ and $\alpha(u)$ are the positive continuous functions on the interval $[0,1], n(u) \in C[0,1]$. The following conclusions will be given: 
Theorem 1. Let $\alpha=\max _{u \in[0,1]} \alpha(u), A=\max _{u \in[0,1]} l(u)$, if $\alpha<1$ and the function $w(u)$ satisfy that

$$
w(u)<\frac{1 / \Gamma(1-\alpha)}{2 A} .
$$

There is only one answer that fits the problem (1).

For another more general case,

$$
\left\{\begin{array}{l}
D^{\alpha^{*}(u)} w(u)=l^{*}(u) w^{2}(u)+m^{*}(u) w(u)+n^{*}(u), \quad u \in(0,1) \\
w(0)=0
\end{array}\right.
$$

where $\alpha^{*}(u)$ and $l^{*}(u)$ are the positive continuous functions on the interval $[0,1], m^{*}(u), n^{*}(u) \in C[0,1]$. We will also give a stronger result as below:

Theorem 2. Let $\alpha^{*}=\max _{u \in[0,1]} \alpha^{*}(u), A^{*}=\max _{u \in[0,1]} l^{*}(u), B^{*}=\max _{u \in[0,1]}$ $\left|m^{*}(u)\right|$, if $\alpha^{*}<1$, and the function $w(u)$ satisfies that

$$
w(u)<\frac{1 / \Gamma\left(1-\alpha^{*}\right)-B^{*}}{2 A^{*}}
$$

There is only one answer that fits the problem (4).

Finally, we will point out an error in the derivation of proving the following theorem and give a correct proof process (that is, Theorem 3 in ref. [4]):

Theorem 3. The following initial value problem.

$$
\left\{\begin{array}{l}
D^{\alpha} w(u)=-q(u) w^{2}(u)-p(u) w(u)+g(u) \\
w(0)=\theta
\end{array}\right.
$$

where $u \in(0,1)$, the constant $\alpha \in(0,1]$, and the functions $p($ $u), q(u), g(u) \in C[0,1]$. Suppose that $M_{p}=\sup _{u \in[0,1]}|p(u)|$, $M_{q}=\sup _{u \in[0,1]}|q(u)|$, and $L$ be the Lipschitz constant of the function $f(w)=w^{2}$, then there is only one solution that meets the problem (6) under the hypothesis $0<M_{p}+M_{q} L / \alpha$ $\Gamma(\alpha)<1$.

The basic framework of this paper would be described as follows: the second section mainly lists the necessary preparatory knowledge; the third and fourth sections, respectively, give the main conclusions (that is, Theorems 1 and 2) and their proof process of this paper; the fifth section points out the error in ref. [4] and corrects it (that is, Theorem 3 as above). The final section gives an example as an application of our main results in this paper.

\section{Preliminary Knowledge}

To prove our main results Theorem 1 and Theorem 2, we first give the following definitions about Caputo fractional derivative.
Definition 4. (see ref. [14]). For a constant $0<\alpha<1$, the $\alpha$ th -order Caputo fractional derivative is described as follows:

$$
D^{\alpha} w(u)=\frac{1}{\Gamma(1-\alpha)} \frac{d}{d u} \int_{0}^{u} \frac{w(s)}{(u-s)^{\alpha}} d s .
$$

Definition 5. (see ref. [11]). For a bounded function $0<\alpha(u$ )$<1$, the $\alpha(u)$ th -order Caputo fractional derivative is described as follows:

$$
D^{\alpha(u)} w(u)=\frac{1}{\Gamma(1-\alpha(u))} \frac{d}{d u} \int_{0}^{u} \frac{w(s)}{(u-s)^{\alpha(u)}} d s
$$

In our recent research results, see ref. [15], the following lemma has been proved by using mathematical methods such as Gronwall-Bellman inequality, which also an important tool to prove the main conclusions of this paper.

Lemma 6. If the function $F(u, s)>0$ is smooth for any $u \in R$, we have

$$
\int_{0}^{u} F(u, s) g(s) d s=0
$$

Then, for any $s \in R$, we can get that $g(s)=0$.

\section{Proof of the First Main Result}

We shall give a very detailed proof process to Theorem 1 in this section. To do this, we suppose that the problem (2) has two different solutions $w_{1}(u)$ and $w_{2}(u)$, that is,

$$
\left\{\begin{array}{l}
D^{\alpha(u)} w_{1}(u)=l(u) w_{1}^{2}(u)+n(u) \\
D^{\alpha(u)} w_{2}(u)=l(u) w_{2}^{2}(u)+n(u)
\end{array}\right.
$$
lows,

By using the Definition 5, we have the equation as fol$\frac{1}{\Gamma(1-\alpha(u))} \frac{d}{d u} \int_{0}^{u}(u-s)^{-\alpha(u)}\left(w_{1}(s)-w_{2}(s)\right) d s=l(u)\left(w_{1}^{2}(u)-w_{2}^{2}(u)\right)$.

Therefore,

$\frac{d}{d u} \int_{0}^{u}(u-s)^{-\alpha(u)}\left(w_{1}(s)-w_{2}(s)\right) d s=\Gamma(1-\alpha(u)) l(u)\left(w_{1}^{2}(u)-w_{2}^{2}(u)\right)$.

Integrating on both sides of the above equality, and according to the initial value condition $w(0)=0$, we get

$$
\int_{0}^{u}(u-s)^{-\alpha(u)}\left(w_{1}(s)-w_{2}(s)\right) d s=\int_{0}^{u} \Gamma(1-\alpha(s)) l(s)\left(w_{1}^{2}(s)-w_{2}^{2}(s)\right) d s .
$$


The above equality obviously implies that

$$
\int_{0}^{u}\left[(u-s)^{-\alpha(u)}-\Gamma(1-\alpha(s)) l(s)\left(w_{1}(s)+w_{2}(s)\right)\right]\left(w_{1}(s)-w_{2}(s)\right) d s=0 .
$$

$$
\begin{gathered}
\text { Since } 0<\alpha=\max _{u \in[0,1]} \alpha(u)<1,0<A=\max _{u \in[0,1]} l(u) \text {, and } \\
w_{i}(s)<\frac{1 / \Gamma(1-\alpha)}{2 A}, i=1,2 .
\end{gathered}
$$

We have a conclusion that for any $s \in(0,1)$,

$$
w_{1}(s)+w_{2}(s) \quad<\frac{1 / \Gamma(1-\alpha)}{A} \leq \frac{1 / \Gamma(1-\alpha(s))}{l(s)} .
$$

Moreover, for any $0<s<u<1,(u-s)^{-\alpha(u)}>1$, which means that

$$
(u-s)^{-\alpha(u)}>\Gamma(1-\alpha(s)) l(s)\left(w_{1}(s)+w_{2}(s)\right) .
$$

Therefore,

$$
(u-s)^{-\alpha(u)}-\Gamma(1-\alpha(s)) l(s)\left(w_{1}(s)+w_{2}(s)\right)>0 .
$$

This, combining with (14) and applying Lemma 6, we have $w_{1}(s)=w_{2}(s)$. This contradiction completes all the proof processes of Theorem 1 .

\section{Proof of the Second Main Result}

We will prove Theorem 2 in this section. Similar to the previous section, we also adopt the idea of counter evidence. Assuming $w_{1}(u)$ and $w_{2}(u)$ are the two solutions of the problem (4), then

$$
\left\{\begin{array}{l}
D^{\alpha^{*}(u)} w_{1}(u)=l^{*}(u) w_{1}^{2}(u)+m^{*}(u) w_{1}(u)+n^{*}(u), \\
D^{\alpha^{*}(u)} w_{2}(u)=l^{*}(u) w_{2}^{2}(u)+m^{*}(u) w_{2}(u)+n^{*}(u) .
\end{array}\right.
$$

Subtracting the above two equalities, and using Definition 5 , we get

$$
\begin{aligned}
& \frac{1}{\Gamma\left(1-\alpha^{*}(u)\right)} \frac{d}{d u} \int_{0}^{u}(u-s)^{-\alpha^{*}(u)}\left(w_{1}(s)-w_{2}(s)\right) d s \\
& \quad=l^{*}(u)\left(w_{1}^{2}(u)-w_{2}^{2}(u)\right)+m^{*}(u)\left(w_{1}(u)-w_{2}(u)\right) .
\end{aligned}
$$

That is,

$$
\begin{aligned}
& \frac{d}{d u} \int_{0}^{u}(u-s)^{-\alpha^{*}(u)}\left(w_{1}(s)-w_{2}(s)\right) d s \\
& \quad=\Gamma\left(1-\alpha^{*}(u)\right)\left[l ^ { * } ( u ) \left(w_{1}^{2}(u)\right.\right. \\
& \left.\left.\quad-w_{2}^{2}(u)\right)+m^{*}(u)\left(w_{1}(u)-w_{2}(u)\right)\right]
\end{aligned}
$$

Performing the similar operation as the previous section, we can get

$$
\begin{aligned}
& \int_{0}^{u}(u-s)^{-\alpha^{*}(u)}\left(w_{1}(s)-w_{2}(s)\right) d s \\
& \quad=\int_{0}^{u} \Gamma\left(1-\alpha^{*}(s)\right)\left[l^{*}(s)\left(w_{1}^{2}(s)-w_{2}^{2}(s)\right)\right. \\
& \left.\quad+m^{*}(s)\left(w_{1}(s)-w_{2}(s)\right)\right] d s .
\end{aligned}
$$

Therefore,

$$
\begin{gathered}
\int_{0}^{u}\left[(u-s)^{-\alpha^{*}(u)}-\Gamma\left(1-\alpha^{*}(s)\right) l^{*}(s)\left(w_{1}(s)+w_{2}(s)\right)\right. \\
\left.-\Gamma\left(1-\alpha^{*}(s)\right) m^{*}(s)\right]\left(w_{1}(s)-w_{2}(s)\right) d s=0 .
\end{gathered}
$$

Since

$$
w_{i}(s)<\frac{1 / \Gamma\left(1-\alpha^{*}\right)-B^{*}}{2 A^{*}}, i=1,2 \text {, }
$$

we have

$$
w_{1}(s)+w_{2}(s)<\frac{1 / \Gamma\left(1-\alpha^{*}(s)\right)-m^{*}(s)}{l^{*}(s)}
$$

The fact $(u-s)^{-\alpha^{*}(u)}>1,0<s<u<1$ including that $(u-s)^{-\alpha^{*}(u)}>1>\Gamma\left(1-\alpha^{*}(s)\right)\left[l^{*}(s)\left(w_{1}(s)+w_{2}(s)\right)+m^{*}(s)\right]$

which means that

$(u-s)^{-\alpha^{*}(u)}-\Gamma\left(1-\alpha^{*}(s)\right)\left[l^{*}(s)\left(w_{1}(s)+w_{2}(s)\right)+m^{*}(s)\right]>0$.

Therefore, we can deduce that $w_{1}(s)=w_{2}(s)$ by applying Lemma 6. This contradiction leads to the result of Theorem 2.

\section{Derivation Error Correction}

In this section, we mainly point out an error in the process of proving Theorem 3 (that is, Theorem 3 in this paper) in ref. [4] (see theorem 3) and correct it. This error mainly appears in lines 12-15 (3.9) on page 284 in ref. [4] which is described as follows:

$$
\begin{aligned}
\left|w_{1}-w_{2}\right| & \leq \frac{1}{\Gamma(\alpha)} \int_{0}^{u} \frac{\left|p(t)\left(w_{2}(t)-w_{1}(t)\right)+q(t)\left(w_{2}^{2}(t)-w_{1}^{2}(t)\right)\right|}{(u-t)^{1-\alpha}} d t \\
& \leq \frac{M_{p}+M_{q} L}{\Gamma(\alpha)}\left|\int_{0}^{u} \frac{1}{(u-t)^{1-\alpha}} d t\right|\left|w_{1}-w_{2}\right| \\
& \leq \frac{M_{p}+M_{q} L}{\Gamma(\alpha)} \frac{u^{\alpha}}{\alpha}\left|w_{1}-w_{2}\right| \leq \frac{M_{p}+M_{q} L}{\alpha \Gamma(\alpha)}\left|w_{1}-w_{2}\right| .
\end{aligned}
$$

This derivation process is problematic, because $\mid w_{1}-$ $w_{2}$ I cannot be extracted from the integral in the second 
inequality. However, the final conclusion is correct. We hereby make the following corrections to the proof process:

Since the continuous functions $w_{1}$ and $w_{2}$ are the solutions of the problem (6), we have the fact that $\max _{v \in[0,1]} \mid w_{1}(v)$ $-w_{2}(v) \mid$ exists, and as a result, for any $u \in[0,1]$,

$$
\begin{aligned}
\left|w_{1}(u)-w_{2}(u)\right| & \leq \frac{1}{\Gamma(\alpha)} \int_{0}^{u} \frac{\left|p(t)\left(w_{2}(t)-w_{1}(t)\right)+q(t)\left(w_{2}^{2}(t)-w_{1}^{2}(t)\right)\right|}{(u-t)^{1-\alpha}} d t \\
& \leq \frac{M_{p}+M_{q} L}{\Gamma(\alpha)} \cdot\left|\int_{0}^{u} \frac{1}{(u-t)^{1-\alpha}} d t\right| \cdot \max _{v \in[0,1]}\left|w_{1}(v)-w_{2}(v)\right| \\
& \leq \frac{M_{p}+M_{q} L}{\Gamma(\alpha)} \cdot \frac{u^{\alpha}}{\alpha} \cdot \max _{v \in[0,1]}\left|w_{1}(v)-w_{2}(v)\right| \\
& \leq \frac{M_{p}+M_{q} L}{\alpha \Gamma(\alpha)} \max _{v \in[0,1]}\left|w_{1}(v)-w_{2}(v)\right|,
\end{aligned}
$$

which means that

$$
\max _{v \in[0,1]}\left|w_{1}(v)-w_{2}(v)\right| \leq \frac{M_{p}+M_{q} L}{\alpha \Gamma(\alpha)} \max _{v \in[0,1]}\left|w_{1}(v)-w_{2}(v)\right| .
$$

Because of $\xi=M_{p}+M_{q} L / \alpha \Gamma(\alpha)<1$, we get

$$
0 \leq \max _{v \in[0,1]}\left|w_{1}(v)-w_{2}(v)\right|(1-\xi) \leq 0 .
$$

So, we have $\max _{v \in[0,1]}\left|w_{1}(v)-w_{2}(v)\right|=0$, which implying the final conclusion $w_{1}=w_{2}$ of this theorem.

\section{Example}

In this section, we will give a simple application of the Theorem 2. Considering the following initial value problem,

$$
\left\{\begin{array}{l}
D^{\alpha^{*}(u)} y(u)=\frac{\sqrt{u}}{8} y^{2}(u)+\frac{1}{5} y(u)+c(u), \quad u \in[0,1] \\
y(0)=0 .
\end{array}\right.
$$

Here,

$$
\left\{\begin{array}{l}
\alpha^{*}(u)=u \cos u, \\
c(u)=u^{q}\left(-\frac{11}{5}-\frac{17}{8} u^{q+1 / 2}+\frac{u^{-\alpha^{*}(u)} \Gamma(1+q)}{\Gamma\left(1+q-\alpha^{*}(u)\right)}\right) .
\end{array}\right.
$$

This is an example which has been first appeared in [11], and its exact solution is $y(u)=u^{q}(q>0)$.

We draw the picture of the function on the $[0,1]$ interval by using MATLAB (See Figure 1) and calculate the maximum value of the function $\alpha^{*}(u)$ on the [0,1] interval. The accuracy is taken to the fourth place after the decimal point, and the approximate value of the function $\alpha^{*}(u)$ is equal to 0.5611 , which is strictly less than 0.57 .

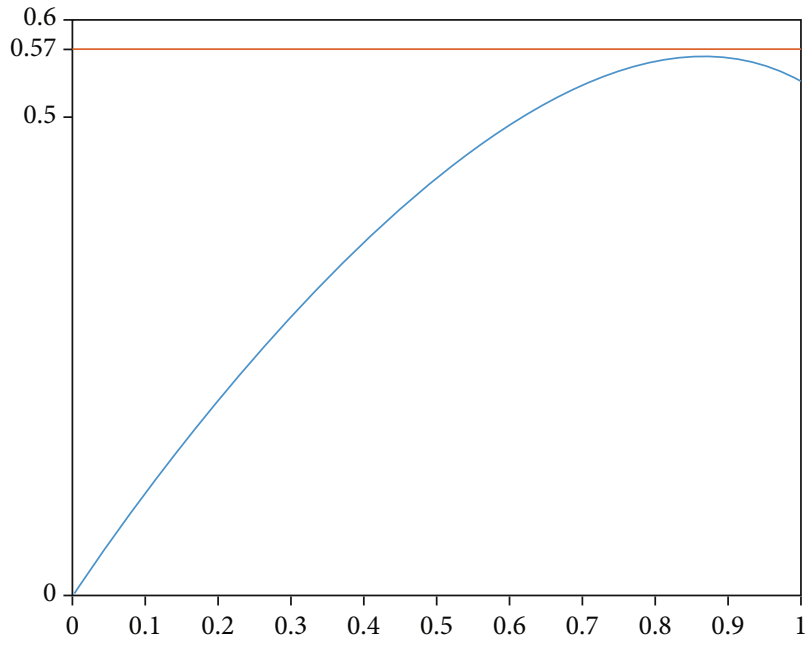

Figure 1: Picture of the function $\alpha^{*}(u)$.

We hence get the following inequality,

$$
\alpha^{*}=\max _{u \in[0,1]} \alpha^{*}(u)<0.57
$$

According to the definition of the $A^{*}, B^{*}$, we can get that

$$
A^{*}=\frac{1}{8}, B^{*}=\frac{1}{5}
$$

Therefore,

$$
\frac{1 / \Gamma\left(1-\alpha^{*}\right)-B^{*}}{2 A^{*}} \geq \frac{1 / \Gamma(1-0.57)-1 / 5}{2 \times 1 / 8} .
$$

Calculating once again by applying MATLAB, and the accuracy is taken to the fourth place after the decimal point. The value on the right of the above inequality is approximately 1.1412 .

Hence,

$$
y(u) \leq 1<\frac{1 / \Gamma\left(1-\alpha^{*}\right)-B^{*}}{2 A^{*}} .
$$

By Theorem 2, the problem (32) has at most one solution.

\section{Data Availability}

All data included in this paper are available upon request by contact with the corresponding author.

\section{Conflicts of Interest}

The authors declare that they have no conflicts of interest.

\section{Authors' Contributions}

Each author has contributed to the completion of this paper. More specifically, supervision was contributed by Shi-you 
Lin (contribution rate: 50\%). Main theorem proving was contributed by Li-sha Chen (contribution rate: $46 \%$ ). Writing-original draft preparation was contributed by Boyang Li (contribution rate: $4 \%$ ).

\section{Acknowledgments}

The authors would like to extend their heartfelt thanks and great honor to the editors and referees for their valuable suggestions on this paper. This work has received the support of some foundations: National Natural Science Foundation of China (Grant no. 11761027), Natural Science Foundation of Hainan Province (Grant no. 2019RC186), and Innovative Scientific Research Projects of Graduate Students in Hainan Province (Grant no. Qhys2021-300).

\section{References}

[1] S. Tsay, P. Gabriel, M. King, and G. L. Stephens, "Spectral reflectance and atmospheric energetics in cirrus-like clouds. Part II: applications of a Fourier-Riccati approach to radiative transfer," Journal of the Atmospheric Sciences, vol. 53, no. 23, pp. 3450-3467, 1996.

[2] M. Silver, R. Joseph, and D. Hoult, "Selective spin inversion in nuclear magnetic resonance and coherent optics through an exact solution of the Bloch-Riccati equation," Physical Review A General Physics, vol. 31, no. 4, pp. 2753-2755, 1985.

[3] K. Nosrati and M. Shafiee, "On the convergence and stability of fractional singular Kalman filter and Riccati equation," Journal of the Franklin Institute, vol. 357, no. 11, pp. 7188-7210, 2020.

[4] B. Kashkari and M. Syam, "Fractional-order Legendre operational matrix of fractional integration for solving the Riccati equation with fractional order," Applied Mathematics and Computation, vol. 290, pp. 281-291, 2016.

[5] M. Khader, "Numerical treatment for solving fractional Riccati differential equation," Differential Equations and Dynamical Systems, vol. 21, no. 1, pp. 32-37, 2013.

[6] R. Yan, M. Han, Q. Ma, and X. Ding, "A spectral collocation method for nonlinear fractional initial value problems with a variable-order fractional derivative," Computational and Applied Mathematics, vol. 38, no. 2, pp. 1-25, 2019.

[7] M. Gulsu, Y. Ozturk, and A. Anapali, "A collocation method for solving fractional Riccati differential equation," Journal of Applied Mathematics, vol. 5, no. 6, 884 pages, 2013.

[8] M. Mehmet, "On the solutions fractional Riccati differential equation with modified Riemann-Liouville derivative," International Journal of Differential Equations, vol. 2012, 17 pages, 2012.

[9] Y. Zhi, "A few kinds of Riccati equation integrable conditions and variable separation method," Applied Mechanics and Materials, vol. 556-562, pp. 3642-3647, 2014.

[10] M. Syam, H. Siyyam, and I. al-Subaihi, "Tau-path following method for solving the Riccati equation with fractional order," Journal of Computational Methods in Physics, vol. 2014, 7 pages, 2014.

[11] E. H. Doha, M. A. Abdelkawy, A. Z. M. Amin, and D. Baleanu, "Approximate solutions for solving nonlinear variable-order fractional Riccati differential equations," Nonlinear Analysis: Modelling and Control, vol. 24, no. 2, pp. 176-188, 2019.
[12] M. Syam and H. Jaradat, "An accurate method for solving Riccati equation with fractional variable-order," Journal of Interpolation and Approximation in Scientific Computing, vol. 2018, no. 1, pp. 1-12, 2018.

[13] D. Tvyordyj, "Hereditary Riccati equation with fractional derivative of variable order," Journal of Mathematical Sciences, vol. 253, no. 4, pp. 564-572, 2021.

[14] A. Kilbas, H. Srivastava, and J. Trujillo, "Theory and applications of fractional differential equations," North-Holland Mathematics Studies, vol. 204, pp. 69-132, 2006.

[15] L. Chen, S. Lin, and B. Li, "Uniqueness of solutions for the initial value problem of a simple type Riccati equation with variable fractional order," in Journal of Physics: Conference Series, Volume 1995, 2021 3rd International Conference on Computer Modeling, Simulation and Algorithm (CMSA2021), vol. 1995, Shanghai, China, 2021. 